\title{
Utilization of Single Whole Body Vibration Training Unit in Rehabilitation of Elderly Patients with Neurological Disorders
}

\author{
Jaroslav Majerník \\ Pavol Jozef Šafárik University in \\ Košice, Trieda SNP 1, 04011 \\ Košice, Slovakia \\ Technical University of Ostrava, \\ 17. listopadu 15, Ostrava - Poruba, \\ Czech Republic \\ Email: jaroslav.majernik@upjs.sk
}

\author{
Miriam Dziaková \\ Louis Pasteur University Hospital \\ in Košice, Trieda SNP 1, 04011 \\ Košice, Slovakia \\ Email: miriam.dziakova@unlp.sk
}

\author{
Jozef Živčák \\ Technical University in Košice, \\ Letná 9, 04200 Košice, Slovakia \\ Email: jozef.zivcak@tuke.sk
}

\begin{abstract}
Whole body vibration (WBV) techniques are increasingly applied in rehabilitation processes to improve patients' functional mobility. Therapeutic usage of WBV reported enhanced muscular strength, power or even bone density. Therefore, the purpose of our study was to investigate immediate response of single WBV training unit on mobility of elderly patients with neurological disorders. Nineteen patients $(66.74 \pm 3.65$ years, 6 males and 13 females) were assessed before WBV (10 min, $30 \mathrm{~Hz}$, vertical $2 \mathrm{~mm}$ ) and then $1 \mathrm{~min}$ afterwards. Individual changes in gait kinematics indicated positive effects of $\mathrm{WBV}$, while kinematics of the gait was more symmetric considering right and left side. Individually, depending on the disease's severity, these changes were more or less significant. The improvements in gait kinematics convinced us that WBV can be carefully used in patients' therapy and it may be used together with individually planed rehabilitation processes to bring more satisfying results.
\end{abstract}

\section{INTRODUCTION}

Ageing of the population brings growing demands on social and health care systems due to the increased risks of various chronic diseases in older individuals. Health related quality of human life and loss of independence can be also affected by natural weakening of human body and its functional systems. This leads to more frequent falls and consecutive health and mobility problems [1-3].

Here, the disorders like sensory loss, vestibular dysfunction, impaired vision, muscular weakness, bone rarefaction or gait disorders are multi-factorial disorders that contribute to vulnerability and frailty of elderly persons. Moreover, elderly falls are usually reasons for additional medical interventions. Motor impairment syndromes are also associated with neurological disorders [4]. This group of clinically heterogeneous diseases causes severe problems in coordination, gait, balance, voluntary muscle control, power and strength $[5,6]$.

Strategies to prevent negative consequences of functional mobility diseases have to be widely discussed, assessed and applied in health care of elderly patients. Such strategies include also rehabilitation techniques and physical exercises. Here, due to the published results the WBV can be considered as reliable and effective tool in rehabilitation and sport medicine $[7,8]$.
Continuing in our previous pilot study [9] we hypothesize that the WBV may stimulate muscle activation in elderly patient with neurological disorders and that application of WBV will result in improvement of quality of their gait. Therefore, the purpose of this study was to investigate whether the single WBV session has any positive effects on gait kinematics.

\section{WHOLE BODY VIBRATION}

Whole body vibration (WBV) has been extensively studied for its dangerous effects on humans, especially when exposed as occupational vibration at high amplitudes and specific frequencies [10-12]. However, WBV is also the concept that was already applied in many studies to confirm benefits for astronauts, athletes, wellness of healthy population, but also patients with various diseases [13-15].

Recent clinical works suggest that low amplitude and low frequency of mechanical stimulation of the human body is a safe and effective way to exercise musculoskeletal structures. The studies realized during past decade indicate that WBV may increase muscle strength, neuromuscular function, bone mass and mineral density [16-19], can be useful in improving physical capacity, cardiorespiratory functions, hormonal production, proprioception, and balance [20-22].

Despite of WBV positive effects presented in almost all related research studies, the authors interpret their results with caution. Also, the underlying mechanisms by which WBV enhance neuromuscular performance vary between studies and are still unclear. Inconsistency in presented results is caused by various training protocols and heterogeneity in study designs. However, several mechanisms should be considered as crucial. Vibration stimulates skeletal muscle by activating a stretch reflex analogous to the tonic vibration reflex. Thus the activated muscle spindle activity causes involuntary reflexive muscle contraction in an attempt to control the vibration imposed muscle length change. WBV also evoke changes in postural control strategy where the vibration stimulus generates postural instability and the body activates particular muscles to maintain body balance. Another mechanism is generated 
when oscillations induce a muscle tuning response in an attempt to minimize the propagation of the vibrations throughout the body. Then, the muscles might activate to prevent further transmission of the vibration stimulus through the body.

There are various vibrating platforms available as commercial products used by many wellness centers and rehabilitation clinics. The main differences are in the type of vibration stimulation, frequency range, and amplitude of vibration [23, 24]. Most of the vibrating platforms vibrate sinusoidally. The subjects stand on the platform which oscillates only vertically or side alternating. Other types of vibrating devices uses two separate platforms to produce vibrations for each foot independently. While commercial usage promotes WBV as attractive and effective alternative to resistance training, the therapeutic applications should carefully consider duration of $\mathrm{WBV}$ exposure, main vibration characteristics as well as their effects on neuromuscular system respecting patients' physical capabilities. The rest periods between vibration trainings may also play a significant role in final WBV effects. The effect of vibration may be also tested using various modeling techniques [25].

\section{MATERIAL AND Methods}

\section{A. Patients}

A group of nineteen elderly patients (age $66.74 \pm 3.65$ years, 6 males and 13 females) with various neurological disorders were included in the study. The inclusion criteria were ambulatory patients that are able to walk independently, had no cardiovascular disease or epilepsy, and had no prior experience in WBV training. All the patients were informed about the WBV training, about the tests to be realized and also about possible risks and benefits of the research. Prior to participation they gave written informed consent approved together with the study design by the University Hospital Ethics Committee.

All participants attended a familiarization session before the study and before the tests were performed. No other physical treatment or intervention was realized at least 24 hour before WBV training session. The anthropometric measures were also taken and registered in patients' experimental protocols. As for the aim of the study, the anthropometric characteristics of lower extremities were preferred, including thigh length (right: $44.89 \pm 2.26 \mathrm{~cm}$, left: $44.42 \pm 2.14 \mathrm{~cm}$ ), calf length (right: $41.89 \pm 3.80 \mathrm{~cm}$, left: $41.58 \pm 3.91 \mathrm{~cm}$ ) and foot length (right: $25.74 \pm 2.82$ $\mathrm{cm}$, left: $25.89 \pm 2.55 \mathrm{~cm})$.

\section{B. Experiment}

The experimental protocol was designed to discover potential immediate response of a single WBV training unit to the quality of gait kinematics in elderly patients. Training sessions were supervised by rehabilitation specialist and measurements as well as WBV sessions were conducted in the same thermally neutral room intended for physical training. All subjects did not engage in any therapeutic or rehabilitation procedures before testing.
The training session started with physical examination and short warming-up walk. Then, the patient's gait was captured and analyzed before WBV exposure. Participants were asked to walk at their natural waking speed along the 6 $\mathrm{m}$ long path. After reaching the end point of the path, they were asked to turn back $\left(180^{\circ}\right)$, i.e. to change the direction of gait, and to walk back to the starting point. Then, they turned back again and walked to the end point of the path, where the last turn back was realized and the patients finished walking in starting point of the path. In that sense, the subjects passed the length of walking path four times. WBV session followed one minute after this control gait was captured and analyzed. Here, each participant stood in static position on the vibration platform (VibroGym inSPORTline) with no shoes and socks and holding on the device handle. Erected posture with slightly bended knees was required during vibration test. The patients were asked to stop the training in the case of any pain responses to vibration. Duration of one WBV training unit was set to $10 \mathrm{~min}$. Sinusoidal vertical vibration frequency was set to $30 \mathrm{~Hz}$ with amplitude of $2 \mathrm{~mm}$. $1 \mathrm{~min}$ rest interval followed after this WBV exposure. Then, the patients walked again and the kinematics of their gait was captured and analyzed in the same way as it was before WBV session. Finally, the kinematics obtained before and after WBV training unit was compared to evaluate its effects on patients' gait.

\section{Parameters}

Gait assessment was performed using our marker-free motion analysis system MAFRAN [26]. The system is based on motion tracking method in video sequence with no passive or active markers attached to the patients' bodies tries to minimize disadvantages of currently available marker-free systems, to bring new of motion analysis and to offer clinicians cheaper, but not worse alternative preserving all advantages of motion analysis.

Here, the patient's gait is captured in sagittal plane using common commercial video camera. Then, the raw record is used in the system to automatically reconstruct motion trajectories of human body anatomical landmarks, i.e. the trajectories of all lower extremity joints and adjacent segments. These trajectories are consequently used to calculate other kinematical parameters for detailed description of patient's gait. Here analyzed parameters include positions, velocities and accelerations of individual joints, hip flexion/extension, knee flexion/extension, and ankle plantar/dorsal flexion angles, gait cycle length, gait cycle time, gait cycle velocity, cadence (cycles per minute), stance phase and swing phase of the gait cycle.

\section{Statistics}

All acquired kinematical parameters were analyzed individually within the patient and then within the group of here included patients as well. The kinematical characteristics of the patients were evaluated as differences between right and left side. The hypothesis was based on assumption that these differences should be smaller after WBV training comparing values obtained before WBV training. Otherwise, the WBV will probably have no 
immediate benefits for gait kinematics. Statistical methods included descriptive statistics and Student's paired t-test and were used to ascertain specific and significant differences. The significance level was set to $\mathrm{P}<0.05$.

\section{RESULTS}

WBV session was well accepted by all elderly patients included in this continuing study. No one felt any pain or expressed any problems during WBV exposure. A first analysis was performed with anatomical joint angles of lower extremities in sagittal plane. Individually, no of the patients had the same curves of all tree joints comparing before and after WBV values. At least one joint's trajectory was changed either in positive or negative direction. An example of anatomical joint angles changes in 65 years old male patient with right hemiparesis is shown on following figures.

Figure 1 shows hip flexion/extension angle before and after single WBV training unit. A paired t-test determined that the mean decrease of differences $(M=0.47, S D=4,29$, $\mathrm{N}=51$ ) was not significantly greater than zero, $\mathrm{t}(50)=0.78$, two-tail $\mathrm{p}=0.441(95 \% \mathrm{CL}=1.208)$, providing evidence that the WBV was not effective in reduction of differences between right and left hip flexion/extension angle.

Figure 2 shows knee flexion/extension angle before and after single WBV training unit. Knee flexion/extension angle of the same patient showed that the mean differences between right and left side before and after WBV were decreased, but the mean decrease of differences $(\mathrm{M}=0.54$, $\mathrm{SD}=8,207, \mathrm{~N}=51$ ) was not significantly greater than zero, $\mathrm{t}(50)=0,47$, two-tail $\mathrm{p}=0.643 \quad(95 \% \mathrm{CL}=2.308)$, providing evidence that the WBV was not effective in reduction of differences between right and left knee flexion/extension angle in this patient.

Figure 3 shows ankle plantar/dorsal flexion angle before and after single WBV training unit. The mean decrease of the right and the left side differences in ankle plantar/dorsal flexion angle of the same 65 years old male patient $(\mathrm{M}=3.79, \mathrm{SD}=4,089, \mathrm{~N}=51)$ was significantly greater than zero, $\mathrm{t}(50)=6.63$, two-tail $\mathrm{p}=0.000 \quad(95 \% \quad \mathrm{CL}=1.150)$ provided evidence that the WBV was effective in reduction of differences between right and left ankle plantar/dorsal flexion angle.

Anatomical joint angles were analyzed in all participants of this study in the same way. The summary of right and lefts side differences in anatomical joint angles in elderly patients showed that the most significant changes were registered in the ankle plantar/dorsal flexion angle (89.47\%), followed by hip flexion/extension angle $(73.68 \%)$ and knee flexion/extension angle (57.89\%). However, these significant changes include both the positive and the negative changes. The only significant positive changes were chiefly in hip flexion/extension angle (63.16\%) followed by the knee flexion/extension angle (47.37\%) and ankle plantar/dorsal flexion angle (47.37\%).

Individually, there were thirteen patients $(68.42 \%)$ who had at least two significant positive changes of these kinematical parameters or they had no significant negative
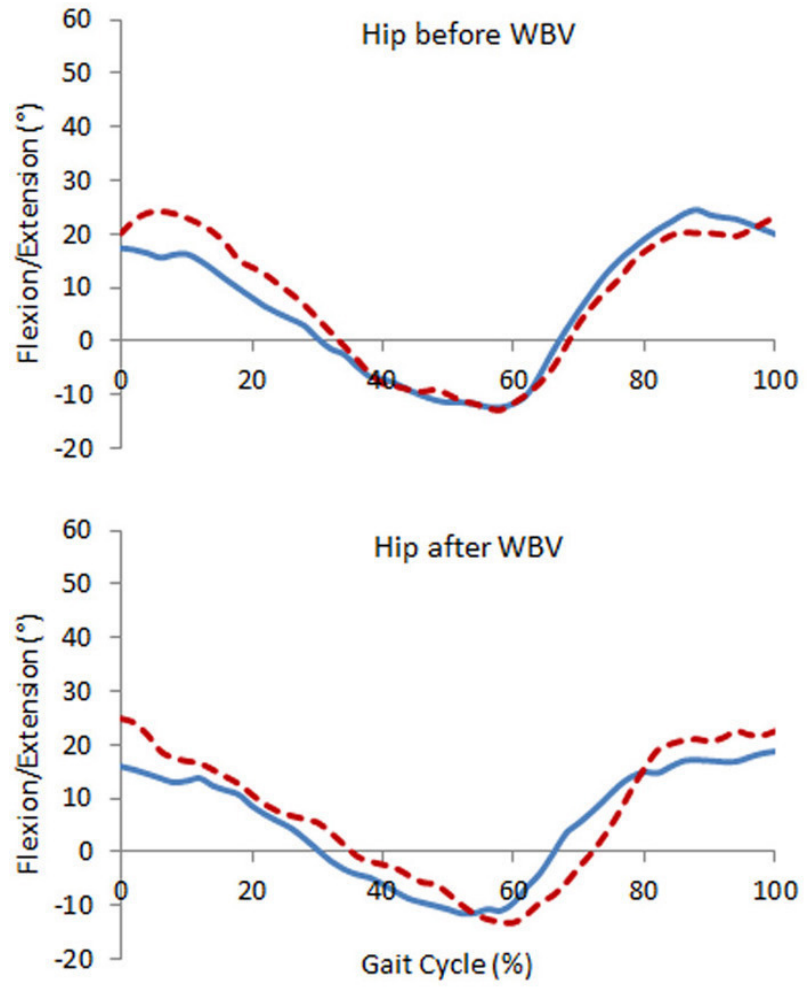

Fig. $1 \mathrm{Hip}$ flexion/extension angle in 65 years old male patient with right hemiparesis before and after WBV exposure (solid line - right leg, dashed line - left leg).
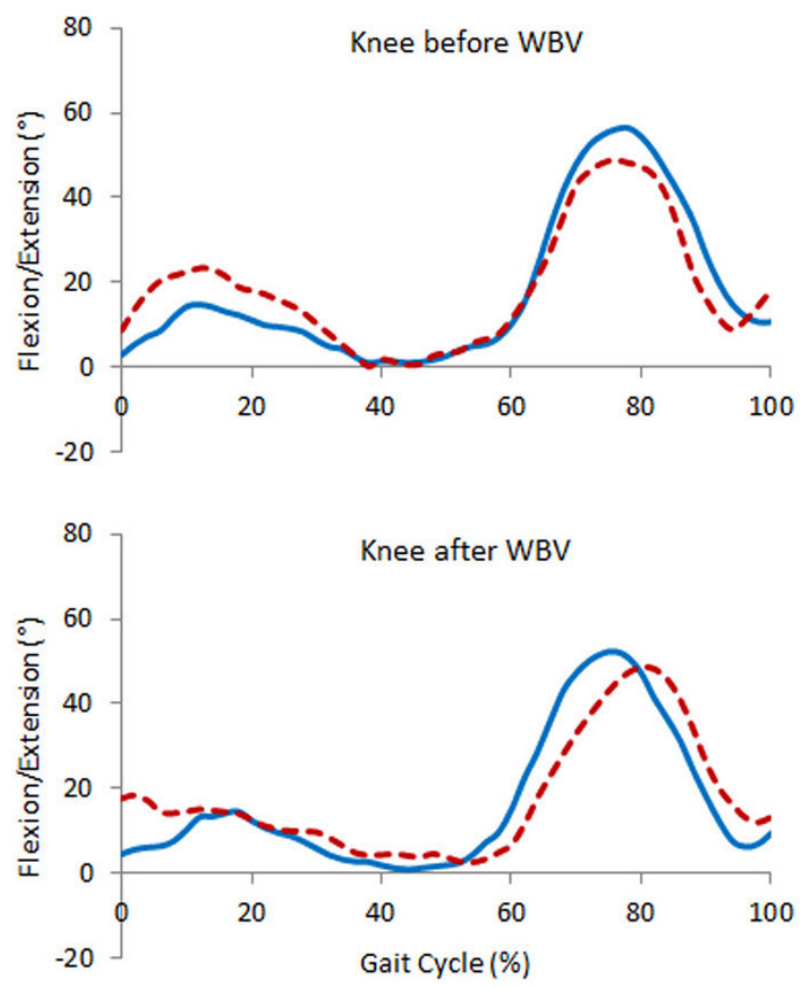

Fig. 2 Knee flexion/extension angle in 65 years old male patient with right hemiparesis before and after WBV exposure (solid line - right leg, dashed line - left leg). 

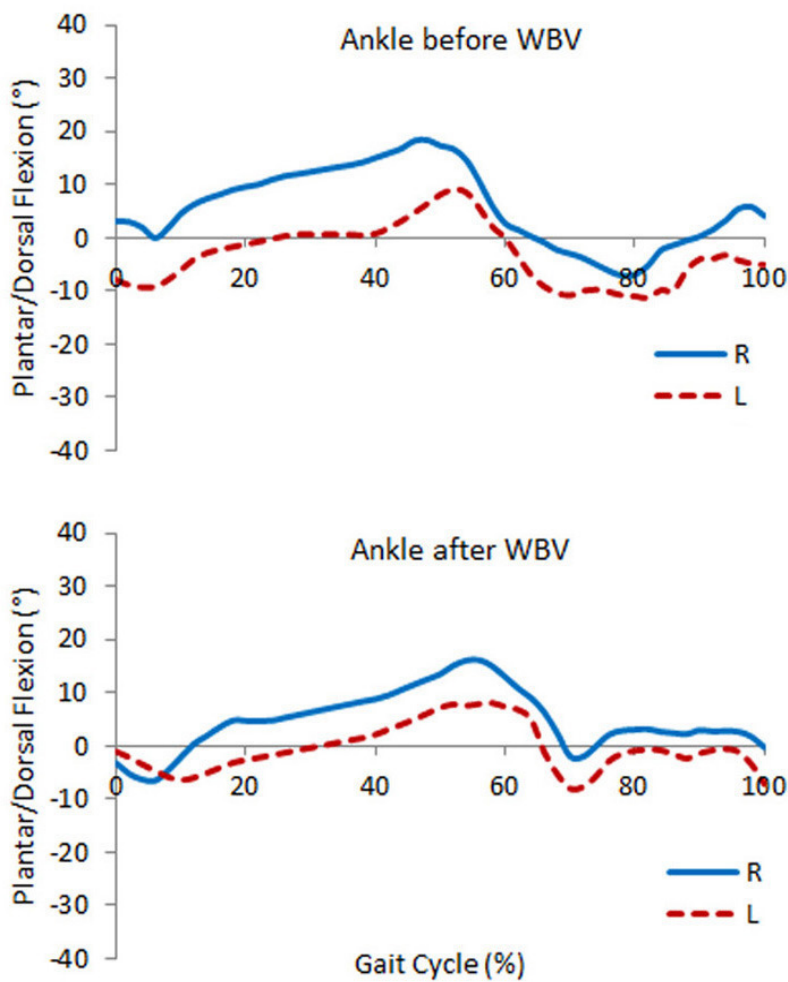

Fig. 3 Ankle plantar/dorsal flexion angle in 65 years old male patient with right hemiparesis before and after WBV exposure (solid line right leg, dashed line - left leg).

changes (subjects 1, 2, 3, 5, 8, 9, 10, 13, 14, 15, 17, 18 and $19)$. Two patients $(10.53 \%)$ had no beneficial improvements resulting from applied WBV exposure (subjects 6 and 12) and four patients $(21.05 \%)$ of the elderly patient subgroup registered worsening because of no significantly positive or only significantly negative changes (subjects 4, 7, 11 and 16).

The second analysis was performed in spatial-temporal parameters. Here, the symmetry of all characteristics was examined and summarized. The mean differences between right and left sides and standard deviations of these parameters are listed in the table 1.

No of here analyzed parameters had significantly neither positive nor negative changes. Nevertheless, some of the parameters had positive and another negative tendency. The positive trends were shown in decreasing differences between right and left side in gait cycle length $(1.470 \pm$ $6.417)$ and velocity $(0.016 \pm 0.056)$. On the other side small increase was registered in cadence $(-0.064 \pm 1.507)$ and gait cycle stance $(-0.885 \pm 4.951$ and swing $(-0.907 \pm 4.932)$ phase. Gait cycle time remained almost unchanged $(0.002 \pm$ $0.043)$.

\section{CONCLUSION}

In this study, the effect of single WBV training unit was tested in elderly patients with neurological disorders including multiple sclerosis, Parkinson disease, cerebral palsy and radiculoneuritis. The significant improvement was confirmed mainly in anatomical joint angles rather than in spatial-temporal parameters. As we expected, the single whole body vibration training unit had only short-time effect as six of the beneficial patients were analyzed one week after the experiment and their kinematics was similar to the pre- exposure status. Utilization of WBV training in patients with neurological disorders may result in benefits for kinematics of human motion, but its significance and mechanism still remains unclear and undiscovered.

The results of this pilot study provided invaluable data for rehabilitation specialists as well as for development of further research programs in physiotherapy. Based on the results we obtained, it was also confirmed that an individual WBV training and supervised functional treatment should be specified for particular patient. Further research should be realized to clarify WBV specific benefits. The various training approaches including standing in different static positions, sitting on chair with legs on vibrating platform or performing exercises on the platform during therapy sessions should be investigated as well.

\section{ACKNOWLEDGMENT}

This work has been elaborated in the framework of the project "Support research and development in the Moravian-Silesian Region 2014 DT 1 - International research teams" (RRC/07/2014), financed from the budget of the Moravian-Silesian Region (50\%) and in the framework of the project KEGA 020UK-4/2014 (50\%).

TABLE I.

SPATIAL-TEMPORAL CHARACTERISTICS OF PATIENTS' GAIT OBTAINED BEFORE (PRE) AND AFTER (POST) WBV EXPOSURE (N=19).

\begin{tabular}{|l|l|l|l|}
\hline \multicolumn{1}{|c|}{ Parameter } & \multicolumn{1}{c|}{ Pre WBV } & \multicolumn{1}{c|}{ Post WBV } & \multicolumn{1}{c|}{ Delta pre/post } \\
\hline GC length $(\mathrm{cm})$ & $5.751 \pm 4.170$ & $4.281 \pm 4.708$ & $1.470 \pm 6.417$ \\
\hline GC time $(\mathrm{s})$ & $0.086 \pm 0.075$ & $0.084 \pm 0.077$ & $0.002 \pm 0.043$ \\
\hline GC velocity $(\mathrm{m} / \mathrm{s})$ & $0.067 \pm 0.038$ & $0.051 \pm 0.048$ & $0.016 \pm 0.056$ \\
\hline Cadence $(\mathrm{GC} / \mathrm{min})$ & $2.014 \pm 1.284$ & $2.078 \pm 1.286$ & $-0.064 \pm 1.507$ \\
\hline Stance phase (\%) & $3.556 \pm 2.944$ & $4.441 \pm 4.984$ & $-0.885 \pm 4.951$ \\
\hline Swing phase (\%) & $3.562 \pm 2.942$ & $4.469 \pm 4.963$ & $-0.907 \pm 4.932$ \\
\hline
\end{tabular}




\section{REFERENCES}

[1] S. Rogan, R. Hilfiker, K. Herren, L. Radlinger, E.D. De Bruin, "Effects of whole-body vibration on postural control in elderly: A systematic review and meta-analysis", BMC Geriatric, 2011, 11, art. no. 72, DOI: 10.1186/1471-2318-11-72.

[2] M. Sitjà-Rabert, M.J. Martínez-Zapata, A. Fort-Vanmeerhaeghe, F. Rey-Abella, D. Romero-Rodríguez, X. Bonfill, X. "Whole body vibration for older persons: an open randomized, multicentre, parallel, clinical trial", BMC geriatrics, 2011, 11, p.89, DOI: 10.1186/14712318-11-89.

[3] N. Shibata, K. Ishimatsu, S. Maeda, S. "Gender difference in subjective response to whole-body vibration under standing posture", International Archives of Occupational and Environmental Health, 2012, 85 (2), pp. 171-179, DOI: 10.1007/s00420-011-0657-0.

[4] B.D. Pozo-Cruz, J.C. Adsuar, J.A. Parraca, J.D. Pozo-Cruz, P.R Olivares, N. Gusi, N. "Using whole-body vibration training in patients affected with common neurological diseases: A systematic literature review", Journal of Alternative and Complementary Medicine, 2012, 18 (1), pp. 29-41, DOI: 10.1089/acm.2010.0691.

[5] R.W.K. Lau, T. Teo, F. Yu, R.C.K. Chung, M.Y.C. Pang, "Effects of whole-body vibration on sensorimotor performance in people with parkinson disease: A systematic review", Physical Therapy, 2011, 91 (2), pp. 198-209, DOI: 10.2522/ptj.20100071.

[6] R.D. Pollock, S. Provan, F.C. Martin, D.J. Newham, "The effects of whole body vibration on balance, joint position sense and cutaneous sensation", European Journal of Applied Physiology, 2011, 111 (12), pp. 3069-3077, DOI: 10.1007/s00421-011-1943-y.

[7] S.A. Moussavi-Najarkola, A. Khavanin, R. Mirzaei, M. Salehnia, M. Akbari, "Effects of whole body vibration on outer hair cells' hearing response to distortion product otoacoustic emissions", In Vitro Cellular and Developmental Biology - Animal, 2012, 48 (5), pp. 276283, DOI: 10.1007/s11626-012-9490-3.

[8] T. Furness, N. Bate, L. Welsh, G. Naughton, C. Lorenzen, C. "Efficacy of a whole-body vibration intervention to effect exercise tolerance and functional performance of the lower limbs of people with chronic obstructive pulmonary disease", BMC Pulmonary Medicine, 2012, 12, art. no. 71, DOI: 10.1186/1471-2466-12-71.

[9] J. Majernik, J. Zivcak, "Effect of Whole Body Vibration on Functional Mobility in Elderly Patients", Acta Mechanica Slovaca, Vol. 17 (3), 2013, ISSN 1335-2393, pp. 64-69.

[10] R. Mani, S. Milosavljevic, S. J. Sullivan, "The effect of occupational whole-body vibration on standing balance: A systematic review", Int. Journal of Industrial Ergonomics, 2010, 40 (6), pp. 698-709, DOI: 10.1016/j.ergon.2010.05.009.

[11] S. Maeda, N. J. Mansfield, N. Shibata, "Evaluation of subjective responses to whole-body vibration exposure: Effect of frequency content", International Journal of Industrial Ergonomics, 2008, 38 (56), pp. 509-515, DOI: 10.1016/j.ergon.2007.08.013.

[12] B. R. Santos, Ch. Lariviere, A. Delisle, A. Plamondon, P.-E. Boileau, D. Imbeau, "A laboratory study to quantify the biomechanical responses to whole-body vibration: The influence on balance, reflex response, muscular activity and fatigue", International Journal of Industrial Ergonomics, 2008, 38 (7-8), pp. 626-639, DOI: 10.1016/j.ergon.2008.01.015.

[13] M. Cardinale, J. Wakeling, "Whole body vibration exercise: are vibrations good for you?", Br J Sports Med, 2005, 39 (9), pp. 585589, DOI:10.1136/bjsm.2005.016857.
[14] K. H. Madou, J. B. Cronin, "The effects of whole body vibration on physical and physiological capability in special populations", Hong Kong Physiotherapy Journal, 2008, 26, pp. 24-38, DOI: 10.1016/S1013-7025(09)70005-3.

[15] R.. D. Prisby, M. Lafage-Proust, L. Malaval, A. Belli, L. Vico, "Effects of whole body vibration on the skeleton and other organ systems in man and animal models: What we know and what we need to know", Ageing Research Reviews, 2008, 7 (4), pp. 319-329, DOI: 10.1016/j.arr.2008.07.004

[16] S.F. Baumbach, M. Fasser, H. Polzer, M. Sieb, M., Regauer, W. Mutschler, M. Schieker, M. Blauth, "Study protocol: The effect of whole body vibration on acute unilateral unstable lateral ankle spraina biphasic randomized controlled trial", BMC Musculoskeletal Disorders, 2013, p22, DOI: 10.1186/1471-2474-14-22.

[17] L. Slatkovska, S.M.H.. Alibhai, J. Beyene, H. Hu, A. Demaras, A.M. Cheung, "Effect of 12 months of whole-body vibration therapy on bone density and structure in postmenopausal women: A randomized trial", Annals of Internal Medicine, 2011, 155 (10), pp. 668-679, DOI: 10.7326/0003-4819-155-10-201111150-00005.

[18] M. Cerny and M. Penhaker, "Wireless Body Sensor Network in Health Maintenance Systems“, Elektronika Ir Elektrotechnika, 2011, (9), pp. 113-116, DOI: 10.5755/j01.eee.115.9.762.

[19] J.O. Totosy de Zepetnek, L.M. Giangregorio, B.C. Craven, "Wholebody vibration as potential intervention for people with low bone mineral density and osteoporosis: A review", Journal of Rehabilitation Research and Development, 2009, 46 (4), pp. 529-542, DOI: 10.1682/JRRD.2008.09.0136.

[20] D. Macura, A. Macurova, A. "Bounded solutions of the nonlinear differential systems", International Journal of Pure and Applied Mathematics, 2011, 70 (5), pp. 755-760.

[21] M. Claerbout, B. Gebara, S. Ilsbroukx, S. Verschueren, K. Peers, P. Van Asch, P. Feys, "Effects of 3 weeks' whole body vibration training on muscle strength and functional mobility in hospitalized persons with multiple sclerosis", Multiple Sclerosis, 2012, 18 (4), pp. 498-505, DOI: $10.1177 / 1352458511423267$.

[22] E.G. Artero, J.C. Espada-Fuentes, J. Argüelles-Cienfuegos, A. Román, P.J. Gómez-López, A. Gutiérrez, "Effect of whole-body vibration and resistance training on knee extensors muscular performance", European Journal of Applied Physiology, 2012, 112 (4), pp. 13711378, DOI: 10.1007/s00421-011-2091-0.

[23] V. Kasik, M. Penhaker, V. Novak, R. Bridzik, J. Krawiec, "User Interactive Biomedical Data Web Services Application", ETechnologies and Networks for Development. vol. 171, J. J. Yonazi, E. Sedoyeka, E. Ariwa, and E. ElQawasmeh, Eds., ed, 2011, pp. 223-237, DOI: $10.1007 / 978-3-642-22729-519$.

[24] C. Milanese, F. Piscitelli, C. Simoni, R. Pugliarello, C. Zancanaro, "Effects of whole-body vibration with or without localized radiofrequency on anthropometry, body composition, and motor performance in young nonobese women", Journal of Alternative and Complementary Medicine, 2012, 18 (1), pp. 69-75, DOI: 10.1089/acm.2010.0324.

[25] A. Sonza, Ch. Maurer, M. Achaval, M. A. Zaro, B. M. Nigg, "Human cutaneous sensors on the sole of the foot: Altered sensitivity and recovery time after whole body vibration", Neuroscience Letters, 2013, 533 (1), pp. 81-85, DOI: 10.1016/j.neulet.2012.11.036.

[26] J. Majerník, "Reconstruction of Human Motion Trajectories to Support Human Gait Analysis in Free Moving Subjects", In: Pancerz,K. and Zaitseva,E.: Computational Intelligence, Medicine and Biology; Studies in Computational Intelligence, 2015, 600, pp. 57-77, DOI: 10.1007/978-3-319-16844-9_4. 